\title{
Philosophiques
}

\section{Deux conditions de la légitimité démocratique}

\section{Juliette Roussin}

Volume 46, numéro 1, printemps 2019

La démocratie entre substance et procédure

URI : https://id.erudit.org/iderudit/1062012ar

DOI : https://doi.org/10.7202/1062012ar

Aller au sommaire du numéro

Éditeur(s)

Société de philosophie du Québec

ISSN

0316-2923 (imprimé)

1492-1391 (numérique)

Découvrir la revue

Citer cet article

Roussin, J. (2019). Deux conditions de la légitimité démocratique. Philosophiques, 46(1), 45-66. https://doi.org/10.7202/1062012ar

\section{Résumé de l'article}

Cet article défend une conception duale de la légitimité démocratique, selon laquelle les décisions et actions politiques, pour valoir comme légitimes d'un point de vue démocratique, doivent présenter certains traits procéduraux et substantiels spécifiques. L'objet de l'article est à la fois de chercher à préciser la nature de ces traits, et d'éclaircir les modalités de leur articulation. La thèse qu'il défend est que, si les composantes procédurales et substantielles des résultats démocratiques correspondent à deux ensembles de conditions également nécessaires, mais non suffisantes, pour que ces résultats et le régime qui les produit puissent être tenus pour légitimes, le respect des exigences procédurales bénéficie néanmoins d'une certaine priorité dans l'estimation de la légitimité démocratique. 


\title{
Deux conditions de la légitimité démocratique
}

\author{
JULIETTE ROUSSIN \\ Chercheuse postdoctorale au Centre de Recherche en Éthique
}

\begin{abstract}
RÉSUMÉ. - Cet article défend une conception duale de la légitimité démocratique, selon laquelle les décisions et actions politiques, pour valoir comme légitimes d'un point de vue démocratique, doivent présenter certains traits procéduraux et substantiels spécifiques. L'objet de l'article est à la fois de chercher à préciser la nature de ces traits, et d'éclaircir les modalités de leur articulation. La thèse qu'il défend est que, si les composantes procédurales et substantielles des résultats démocratiques correspondent à deux ensembles de conditions également nécessaires, mais non suffisantes, pour que ces résultats et le régime qui les produit puissent être tenus pour légitimes, le respect des exigences procédurales bénéficie néanmoins d'une certaine priorité dans l'estimation de la légitimité démocratique.
\end{abstract}

ABSTRACT. - In this paper I argue for a dual conception of democratic legitimacy. According to this conception, political decisions and actions must have specific procedural and substantive features to be democratically legitimate. The aim of the paper is both to seek to specify the nature of these features, and to clarify how they can be articulated together. I show that the procedural and substantive components of democratic outcomes correspond to two sets of equally necessary, but not individually sufficient, conditions for democratic legitimacy. I then proceed to argue that respect for procedural requirements should nevertheless be granted a certain priority in the evaluation of democratic legitimacy.

Qu'est-ce qui rend la démocratie légitime ? ${ }^{1}$ La qualité de ses politiques, ou la manière dont elles sont arrêtées ? Comme face à tout dilemme trop simple, on est tenté de répondre: «Les deux!». C'est de fait la voie choisie par cet article, qui s'attache à défendre une conception duale de la légitimité démocratique. Selon cette conception, la légitimité des décisions collectives en démocratie comprend nécessairement deux composantes, procédurale et substantielle. La démocratie est légitime, autrement dit, à la fois en vertu des procédures particulières qui sont employées pour prendre les décisions politiques, et de la teneur de ces décisions.

Mais cette réponse, bien sûr, se heurte presque immédiatement à un dilemme autrement plus complexe. Car la coïncidence des bons procédés et

1. J'ai eu la chance de pouvoir présenter différentes versions de cet article, lors du colloque «Démocratie: entre procédure et substance» organisé par Magali Bessone et Didier Mineur à Rennes I et Science Po Rennes en mars 20I7, et au séminaire de philosophie politique normative de l'EHESS en janvier 20I8. Je souhaiterais remercier leurs organisateurs respectifs, ainsi que les participant.e.s pour leurs questions et commentaires. Je suis en particulier reconnaissante à Charles Girard, Bernard Manin et Nick Alvarez pour leur discussion serrée de certains arguments du texte.

PHILOSOPHIQUES 46/1 — Printemps 2019, p. 45-66 
des bonnes décisions n'a rien de nécessaire. A priori, rien ne semble même particulièrement la favoriser en démocratie ${ }^{2}$. Dès que l'on essaie de tenir ensemble les deux composantes, la possibilité de leur conflit émerge. Ce conflit renvoie à la tension générale entre l'exigence d'une communauté gouvernée par des lois consenties, dont les citoyens peuvent s'estimer les auteurs, et celle d'une communauté gouvernée par des lois valides ou justes, dont on peut considérer qu'elles le sont indépendamment de l'approbation que leur marquent, ou non, les membres de la communauté. Dans le champ démocratique, elle s'incarne symptomatiquement dans la possibilité toujours ouverte d'une opposition entre nombre et raison, majorité et validité. Si les deux éléments doivent être réunis pour que la démocratie soit légitime, que se passe-t-il quand une décision qui a été prise selon la procédure adaptée est d'une qualité médiocre, voire est carrément infâme? Inversement, que penser des décisions politiques qui contournent les procédures habituelles de prise de décision collective, mais pour instituer ou préserver un ordre juste? Ces questions difficiles font ressortir l'attrait potentiel de conceptions monistes ou exclusives de la légitimité démocratique qui, situant le tout de la légitimité dans le respect des procédures ou dans la qualité des décisions politiques, n'ont pas à se préoccuper d'arbitrer entre ces exigences rivales.

C'est pourquoi cet article entreprend, dans un premier temps, de montrer que les conceptions monistes, exclusivement procédurales ou substantielles, débouchent sur des impossibilités logiques qui ne laissent d'autre choix que d'adopter une conception duale de la légitimité démocratique. Le second moment se consacre à l'examen positif de cette conception et cherche à déterminer ce que pourrait être une articulation stable entre ses deux composantes procédurale et substantielle. Il suggère que, pour les concilier d'une façon qui soit tenable, c'est-à-dire qui permette de régler les conflits qui affleurent régulièrement entre leurs exigences, il faut malgré tout disposer d'une règle de priorité entre elles. Autrement dit, si les deux exigences doivent nécessairement être satisfaites pour qu'on puisse parler d'une démocratie légitime, il peut arriver, ponctuellement, que l'une vienne à manquer sans que cela remette en cause la légitimité du régime.

\section{L'impossibilité des approches exclusives de la démocratie}

Le premier moment de l'argument en faveur d'une conception duale de la légitimité démocratique est négatif. Il consiste à mettre en évidence l'irrecevabilité des conceptions monistes ou exclusives de la légitimité démocratique. Plus précisément, il montre qu'il est également impossible de réduire la démocratie à une pure procédure de choix ou à un ensemble substantiel de principes politiques prédéfinis; et qu'il n'est pas non plus concevable que

2. Même les justifications «épistémiques» de la démocratie, dont il sera question plus loin, s'appuient sur un certain nombre de conditions préalables pour fonder l'espoir d'une telle coïncidence régulière. 
les décisions démocratiques tirent entièrement leur légitimité de leur substance ou de la procédure qui a conduit à leur adoption. Les objections aux approches exclusives de la légitimité démocratique sont habituellement d'ordre moral, et consistent à souligner que ces approches ne satisfont pas complètement aux exigences générales de la légitimité politique. La stratégie poursuivie dans cette partie est différente: elle s'appuie sur des considérations logiques pour démontrer l'impossibilité respective des conceptions monistes de la légitimité démocratique. L'argument général est qu'il est tout aussi contradictoire de comprendre la démocratie comme une pure procédure, vierge de tout engagement substantiel, que de l'identifier à un ensemble de valeurs politiques fixées au départ.

\subsection{Approches purement procédurales}

D'après les approches purement procédurales de la démocratie, celle-ci se définit exclusivement comme une procédure particulière de choix collectif. (Par «choix collectif ", "décision collective » ou "décision commune», on entend ici un choix ou une décision politique qui s'applique à tous les membres de la communauté politique.) Elle diffère alors essentiellement des autres régimes par la façon spécifique dont y sont prises les décisions politiques. Qu'est-ce qui fait d'une procédure de choix collectif une procédure proprement démocratique? Admettons pour l'instant qu'une procédure est démocratique si elle est inclusive, participative et égalitaire, c'est-à-dire si elle confère à tous les membres d'une communauté politique donnée un égal pouvoir de contrôle sur les décisions communes. La démocratie se distinguerait donc, selon ces approches, par le caractère inclusif de ses procédures de décision collective et par l'égalité et l'impact politiques effectifs que ces procédures garantissent aux participants, en tant qu'ils ont à la fois la possibilité régulière de concourir aux décisions communes de façon significative, et les moyens d'exercer une influence et une surveillance réelles sur leur élaborations.

Certaines approches purement procédurales de la démocratie défendent également une conception purement procédurale de la légitimité démocratique. Qu'une décision collective soit prise selon une procédure inclusive, égalitaire et participative suffit alors à la désigner non seulement comme démocratique (selon la définition procédurale de la démocratie), mais encore comme pleinement légitime $e^{3}$. Le pur procéduralisme de Jeremy Waldron est de cette sorte. Waldron identifie la démocratie à l'emploi de procédures par-

3. Cette association, caractéristique de ce que l'on peut appeler le "pur procéduralisme", n'est pas systématique. Certains théoriciens définissent la démocratie comme une procédure de décision, mais considèrent que les décisions démocratiques tirent leur légitimité de leur conformité à des critères extérieurs, indépendants de la procédure, tels que les droits de l'homme, l'efficacité économique ou la justice sociale. C'est par exemple la position de Joseph Schumpeter (Capitalisme, socialisme et démocratie, Paris, Payot, I990, p. 356, 319-320) ou de Friedrich Hayek (La constitution de la liberté, Paris, Litec, I994, p. I02, II4, I78). 
ticulières de décision politique, l'élection des représentants au suffrage universel égal et le vote des lois par l'assemblée représentative, dans les deux cas à la majorité des voix, et montre que ces procédures, qui distinguent la démocratie des autres régimes, sont aussi ce qui lui confère sa légitimité propre et la désigne comme un régime supérieur aux autres ${ }^{4}$. Ce qui tout à la fois définit la démocratie et la rend légitime, dans la philosophie politique de Waldron, c'est le respect qu'elle témoigne, par le choix de ses procédures de décision, à l'égalité fondamentale des individus face à la décision politique, dans un contexte de désaccord profond et récurrent sur la meilleure façon de conduire leurs affaires communes ${ }^{5}$.

Selon la conception purement procédurale de la légitimité démocratique, la teneur des décisions politiques n'entre pas en ligne de compte dans l'évaluation de leur légitimité. La décision de réduire drastiquement le taux de l'impôt sur les sociétés est aussi légitime que celle de le multiplier par deux, quels qu'en soient le contexte et les conséquences, tant qu'elle résulte de procédures de choix qui correspondent aux critères généraux d'une procédure démocratique. Une décision collective est légitime si et seulement si elle résulte de l'application d'une procédure démocratique de choix: la question de la justification ou de la justice de la décision est totalement mise de côté lorsqu'il s'agit d'apprécier sa légitimité démocratique ${ }^{6}$.

L'avantage évident d'une telle conception est qu'elle semble faire l'économie de tout présupposé substantiel pour définir la démocratie et ses critères de légitimité. Waldron insiste fortement sur ce point : il est indispensable de séparer la considération de la justice ou de la justification des décisions de celle de leur légitimité, sous peine de reproduire ad infinitum les désaccords politiques qui divisent la communauté démocratique, et qui rendent précisément l'adoption d'une règle de décision nécessaire au départ ${ }^{7}$. De façon comparable, en rapportant la démocratie à une forme de décision, le pur procéduralisme s'épargne potentiellement toute prise de parti contestable sur ce qui vaut ou non comme "démocratique». La liberté de circulation ou l'égalité économique, par exemple, sont-elles démocratiques? Ces questions ne sont pas a priori résolues par la conception procédurale, d'après laquelle la démocratie est d'abord ce que ses acteurs veulent en faire. C'est un régime à la définition ouverte, une construction collective dont les fondations ne sont pas posées par un Législateur extérieur, un groupe d'experts ou

4. Law and Disagreement, Oxford, Clarendon Press, I999, p. 53-54.

5. Ibid., p. II 4 .

6. Nous reprenons ici la distinction tracée par A. J. Simmons ( Justification and Legitimacy", Ethics, vol. I09, n 4, I999, p. 739-77I) et suivie par Waldron ("Rights and Majorities: Rousseau Revisited", in Liberal Rights: Collected Papers, 1981-1991, Cambridge, Cambridge University Press, I993, p. 392-42I), entre justification et légitimité. Une décision est justifiée par son contenu; elle est légitime quand elle émane de l'autorité appropriée.

7. "Rights and Majorities: Rousseau Revisited", p. 393; "A Right-Based Critique of Constitutional Rights ", Oxford Journal of Legal Studies, vol. I3, nº I, mars I993, p. 32. 
une équipe de juristes qui auraient été appelés (et par qui ?) à lui donner préalablement ses orientations et ses limites.

L'approche purement procédurale de la démocratie s'est historiquement heurtée à des critiques normatives massives, au premier rang desquelles l'accusation de n'offrir aucune garantie au régime démocratique contre le risque de tyrannie de la majorité ${ }^{8}$. Couplée à une conception exclusivement procédurale de la légitimité, cette approche n'offre, en effet, pas même la possibilité de dénoncer la tyrannie majoritaire comme inacceptable au regard des critères de la légitimité démocratique. Plus récemment, certains philosophes ont souligné la pauvreté conceptuelle, l'insuffisance théorique d'une approche qui voudrait réduire tout un régime politique à la simple mise en œuvre de certaines règles de décision ${ }^{9}$. Selon ces critiques, la démocratie renvoie à un système politique et social complexe qui ne saurait se ramener à un ensemble de procédures, mais repose au contraire sur un socle de "principes communs ${ }^{10}$ » et une "culture» démocratique partagée ${ }^{11}$. La force de ces différentes objections leur vient de ce qu'elles se fondent sur un appel au sens commun: elles identifient un trait que l'on associe assez naturellement à l'idée de démocratie (un type particulier de société) ou de légitimité (l'absence de tyrannie) et montrent que le pur procéduralisme, ou bien ne permet pas de rendre compte de ce trait, ou bien le tient pour négligeable. Elles en concluent que la conception procédurale est défaillante, ou paradoxale: en aucun cas elle ne mérite qu'on s'y attarde.

Quel que soit leur bien-fondé, c'est aussi la limite de ces objections que de présupposer acquis ce qu'il faudrait prouver, à savoir que la légitimité d'une démocratie dépend au moins pour une part de la teneur des mesures politiques qu'elle met en œuvre et des droits qu'elle protège, ou que la culture démocratique précède le mode de décision démocratique et lui donne sens. Un pur procéduraliste pourrait recevoir ces objections en réitérant simplement ses propres prémisses. Il pourrait arguer, par exemple, qu'il est concevable que l'ethos démocratique procède intégralement de l'habitude de

8. Alexander Hamilton, John Jay, et James Madison, Le fédéraliste, Paris, Garnier, 20I 2 $\mathrm{n}^{\circ}$ Io; John Stuart Mill, De la liberté, Paris, Gallimard, I990, p. 66; Samuel Freeman, "Constitutional Democracy and the Legitimacy of Judicial Review ", Law and Philosophy, vol. 9, $\mathrm{n}^{\circ}$ 4, décembre I990, p. 340; Philip Pettit, Républicanisme: Une théorie de la liberté et du gouvernement, Paris, Gallimard, 2004, p. 26, 89, 239.

9. Cornelius Castoriadis, La montée de l'insignifiance, Paris, Seuil, 2007, p. 267, 28I ; Elizabeth Anderson, "Democracy: Instrumental vs. Non-Instumental Values", in Thomas Christiano, et John Christman, dir., Contemporary Debates in Political Philosophy, Malden, Wiley-Blackwell, 2009, p. 2I 4 .

10. Vincent Descombes, Le raisonnement de l'ours, et autres essais de philosophie pratique, Paris, Seuil, 2007, p. 247.

11. John Rawls, Libéralisme politique, Paris, Presses Universitaires de France, 2006, p. 27, 32, 34, 38; Jürgen Habermas, "La souveraineté populaire comme procédure", in Charles Girard et Alice Le Goff, dir., La démocratie délibérative. Anthologie de textes fondamentaux, Paris, Hermann, 2010, p. I95. 
prendre des décisions de façon démocratique. Ou que l'accusation de «tyrannie majoritaire» cache bien souvent le dépit de la minorité de se voir imposer des mesures qu'elle désapprouve ${ }^{12}$, comme une augmentation de l'impôt sur les sociétés ou l'autorisation du mariage gay. Ou encore, que des procédures inclusives, participatives et égalitaires supposent qu'une place suffisante soit accordée, au cours du processus de décision collective, à la délibération, à la réflexivité, à l'expression de l'opposition, aux mécanismes de contrôle et de révision, et que ces garanties procédurales suffisent à considérer que les décisions qui résultent de ces procédures sont pleinement légitimes $^{13}$. Rien dans ces objections, en d'autres termes, ne contraint le procéduraliste à abandonner sa conception pour reconnaître que des éléments substantiels doivent être pris en considération dans l'évaluation de la légitimité démocratique.

C’est pourquoi, pour établir la nécessité d'une conception duale de la légitimité démocratique, une stratégie plus radicale consiste à faire valoir qu'il n'est en réalité pas possible, d'un strict point de vue logique, de défendre une conception purement procédurale de la démocratie et de la légitimité. L'objection fondamentale que l'on peut opposer au pur procéduralisme est l'incohérence de sa position: alors même qu'il prétend fonder la démocratie et légitimer les décisions politiques qui y sont prises sur une base vierge de tout engagement substantiel, il définit une procédure de décision qui, pour valoir comme démocratique, doit nécessairement satisfaire certains réquisits substantiels. Énoncé de façon synthétique, l'argument de l'impossibilité logique est donc qu'il n'existe pas de pure procédure démocratique ni de décision démocratique dont la légitimité ne soit que procédurale, car tout choix d'une méthode démocratique suppose d'accorder de la valeur à certains principes politiques, que cette méthode a précisément pour vocation de respecter ou de réaliser.

Pour comprendre en quoi le pur procéduralisme peut être tributaire d'engagements substantiels sans paraître manifester de partialité envers certains résultats démocratiques, il importe dans un premier temps de distinguer entre les valeurs qui sont au principe de la procédure et celles qui en résultent. L'engagement substantiel peut en effet se manifester à deux moments distincts du processus démocratique : ex post, au moment d'évaluer la teneur des résultats et leur conformité à des exigences démocratiques substantielles; ou ex ante, au moment de construire la procédure en fonction de certains principes qui se révèlent, à l'examen, foncièrement substan-

12. Jeremy Waldron, "The Core of the Case Against Judicial Review ", Yale Law Journal, vol. II 5, 2005, p. I346-I 406.

13. Richard Bellamy, Political Constitutionalism: A Republican Defence of the Constitutionality of Democracy, Cambridge, Cambridge University Press, 2007. 
tiels. David Estlund ${ }^{14}$ souligne ainsi que, même si les démocrates procéduralistes refusent de reconnaître le moindre critère de ce qui constitue en substance une bonne décision politique, ils ne peuvent (et ne veulent) pas se passer de critères concernant la manière dont les décisions politiques doivent être prises.

Estlund propose de démontrer ce point au moyen d'un raisonnement par l'absurde: en réfléchissant à ce que serait une procédure absolument pure de tout engagement substantiel, on peut montrer qu'elle n'aurait pas les qualités requises d'une règle de choix, et encore moins d'une règle démocratique. Par là, il entend établir que, contrairement à ce qu'avance le pur procéduraliste, "l'équité » (c'est-à-dire le fait de traiter tous les participants de façon rigoureusement égale) ne suffit ni à conférer leur légitimité aux décisions démocratiques ni à rendre compte des traits essentiels de la procédure démocratique de décision. La "portée morale» de ce principe procédural est «trop faible» pour cela ${ }^{15}$. Ce n'est donc que l'ajout de valeurs «extra-procédurales ", substantielles, qui conduit le procéduraliste à défendre les procédures démocratiques et à tenir leurs résultats pour légitimes ${ }^{16}$. L'argument d'Estlund est que l'équité ne permet notamment pas de rendre compte du caractère "agrégatif» de la procédure, c'est-à-dire de sa sensibilité aux préférences individuelles exprimées. Cette propriété correspond à ce que la théorie du choix social appelle la «réponse positive ${ }^{17}$ » : la procédure répond positivement aux préférences individuelles de sorte que, si elles varient, alors la décision collective varie en fonction d'elles. Si la procédure était absolument équitable, selon Estlund, elle ne pourrait pas répondre positivement aux variations des préférences individuelles, car une telle sensibilité aux choix des individus constituerait une rupture de l'équité. Celle-ci requerrait d'après lui un "anonymat complet ${ }^{18}$ » : afin de traiter toutes les personnes de la même façon, il faudrait non seulement que leur identité n'influe pas sur le résultat, mais encore que leurs préférences ne soient pas connues. À partir de là, Estlund peut conclure à l'absurdité du pur procéduralisme: poussé à son terme, il requiert une règle de décision qui, pour être équitable, ne peut agréger que des bulletins vierges. A contrario, s'il veut tenir compte des préférences individuelles, le procéduralisme doit se renier lui-même: dès lors qu'elle se montre sensible aux différentes préférences, la

14. L'autorité de la démocratie: une perspective philosophique, Paris, Hermann, 20I I, p. I6I.

15. Ibid., p. I24.

16. En l'occurrence, ce serait la conviction (substantielle) que les individus, en groupe, sont capables de prendre des décisions politiques rationnelles qui justifierait, selon Estlund, que les États adoptent des procédures démocratiques de décision. Ibid., p. 429.

17. Voir Kenneth O. May, "A Set of Independent Necessary and Sufficient Conditions for Simple Majority Decision ", Econometrica, vol. 20, n 4, octobre I952, p. 680-684.

18. L'autorité de la démocratie, p. I46. 
procédure se soumet à un critère non procédural, qui rogne sur les prérogatives de l'équité par la levée partielle de la condition d'anonymat ${ }^{19}$.

Estlund cherche bien à démontrer l'impossibilité logique du procéduralisme, mais il s'appuie pour cela sur une conception de l'équité qui semble assez contestable. Sa définition de l'anonymat, pour commencer, ne concorde pas avec le sens usuel donné à ce terme. L'anonymat ne requiert pas de gommer tout ce qui caractérise la personne, mais seulement ce qui permettrait de l'identifier. De même, l'équité n'exige pas qu'on adopte une attitude absolument neutre à l'égard de toutes les différences entre les personnes, mais simplement qu'on ne manifeste aucune partialité envers celles qui ne sont pas pertinentes pour juger de l'affaire ${ }^{20}$. Dans le cas du vote, l'équité oblige à traiter toutes les préférences individuelles à égalité, quelles que soient les personnes qui les expriment. Cela implique effectivement d'anonymiser les votes, mais certainement pas de censurer les préférences politiques. Empêcher leur expression au nom de l'équité démocratique a autant de sens que d'interdire aux parties d'un procès de faire valoir leurs arguments sous prétexte de permettre au juge de statuer équitablement sur le litige. L'équité n'exige que l'exclusion des considérations non pertinentes; on voit mal comment les préférences politiques des citoyens pourraient ne pas l'être, lorsqu'il s'agit de prendre une décision collective sur les affaires de la communauté.

On peut se demander, au delà, ce qu'Estlund veut dire exactement lorsqu'il affirme que l'équité est un "critère intrinsèquement procédural ${ }^{21}$ ". En reprenant la distinction entre principes (ex ante) et résultats (expost) des procédures démocratiques, on peut en effet considérer que l'exigence de traiter les participants à la procédure de choix de façon absolument égale résulte, au contraire, de convictions substantielles profonde. Elle s'ancre, entre autres, dans la reconnaissance du statut égal des personnes en tant qu'agents rationnels et moraux, capables de décider pour eux-mêmes des affaires qui les concernent. En l'occurence, le lien de fondation entre procédure démocratique et égalité des personnes est maintes fois énoncé par Waldron, qui en fait le cœur de son argument en faveur de la règle de majorité. C'est de l'égalité de statut qu'il dérive le droit de participation, l'importance morale de la pluralité et l'exigence d'inclusion. Or, même en admettant qu'elle soit «invraisemblablement étroite ${ }^{22}$ », cette notion d'égalité n'est pas purement procédurale, mais suppose une certaine conception de la personne, de ses capacités et de ses droits. Autrement dit, le choix des procédures démocratiques dépend de certains principes qui sont, à l'examen,

19. Ibid., p. I 54.

20. Voir Charles R. Beitz, Political Equality: An Essay in Democratic Theory, Princeton, Princeton University Press, I989, p. 89.

21. L'autorité de la démocratie, p. I35.

22. Beitz, Political Equality, p. 64. 
foncièrement substantiels. Pour démontrer la contradiction logique que recèle le pur procéduralisme, une stratégie plus simple et plus directe que celle d'Estlund consiste donc à débusquer, au cœur de la procédure démocratique, les engagements substantiels qui la fondent.

On voit mal comment le choix d'une procédure pourrait échapper à l'arbitraire tout en ne s'appuyant sur aucune considération de fond. Il se trouve forcément toujours des raisons pour adopter une procédure plutôt qu'une autre. Rien dans l'idée de procédure n'implique que les participants soient comptés également, ni même qu'il y ait des participants. Si l'on choisit d'arrêter les décisions collectives par un vote à la majorité plutôt qu'en les confiant à un seul ou au sort, par exemple, c'est parce que cette méthode incarne et respecte bien mieux, dans son principe, la conviction profonde que la participation égale de tous à la détermination des affaires communes est moralement et politiquement importante. Une procédure n'est qu'une méthode ou un système de règles définissant la manière dont il faut procéder afin de parvenir à un certain résultat - ici, une décision qui oblige l'ensemble. La teneur de ces règles est nécessairement fixée par référence à certains principes indépendants, qu'on estime valables (parce que fonctionnels, ou justes), et qui viennent informer la procédure, en définir le déroulement légitime. La règle de majorité, par exemple, est le produit de considérations pragmatiques - simplicité et clarté du vote majoritaire — et de considérations morales ou de justice - en tant qu'agents moraux égaux, il convient que les citoyens prennent une part égale à la décision qui les lie. Plus généralement, sans la reconnaissance préalable de ce qu'a d'indispensable le principe d'autodétermination inclusive et égale pour le respect de la capacité égale de jugement et d'action des personnes en tant qu'agents rationnels et moraux égaux, il n'y a aucune raison pour que la procédure de décision assume une forme équitable et universelle. En ce sens, comme l'observe Michael Walzer, «aucun dispositif procédural ne peut se justifier sans recourir à un argument substantiel ${ }^{23}$ ».

Cela signifie, plus profondément, que le procéduralisme fait fausse route quand il affirme que la démocratie se définit par un type particulier de procédures de décision. Les remarques qui précèdent indiquent clairement, au contraire, que le choix de certaines procédures de préférence à d'autres est dicté par des principes substantiels (l'égalité de statut, l'agentivité morale) qui justifient aussi qu'on qualifie ces procédures de démocratiques. Autrement dit, ce ne sont pas les procédures, mais les idéaux qu'elles présupposent qui confèrent sa signification, et sa légitimité, à la démocratie. Pour valoir comme démocratique, toute procédure de décision doit nécessaire-

23. «Philosophy and Democracy», Political Theory, vol. 9, n 3, I98I, p. 386; voir également Ronald M. Dworkin, Une question de principe, Paris, Presses Universitaires de France, I996, p. 89; Corey Brettschneider, Les droits du peuple: valeurs de la démocratie, Paris, Hermann, 2009, p. 35-36. 
ment satisfaire certains réquisits substantiels (l'inclusion, l'égalité, la participation) qui dérivent eux-mêmes d'une conception morale fondamentale de la personne humaine comme agent moral égal. Ces valeurs et principes sont ce qui définit la démocratie en propre, non de quelconques procédures; le choix de celles-ci est nécessairement dérivé.

Cela signifie également que la thèse procéduraliste selon laquelle la démocratie pourrait tirer toute sa substance d'elle-même, au gré d'un processus d'autodétermination rendu possible par une procédure de choix pure de tout présupposé substantiel, est une contradiction dans les termes. Contrairement à ce qui peut se passer pour d'autres principes politiques, l'égalité, la participation et l'inclusion ne sont pas devenues des valeurs démocratiques en vertu de l'adhésion exprimée par une majorité de citoyens via leur procédure de décision collective, égalitaire et inclusive. Elles préexistent nécessairement à la procédure démocratique et en structurent les traits. Les procéduralistes commettent donc un contresens lorsqu'ils invoquent la participation inclusive et égale pour justifier de laisser la détermination des valeurs collectives à l'entière discrétion des citoyens. Car du seul fait qu'ils l'invoquent, ils lui reconnaissent le statut de valeur fondamentale pour la démocratie, et contredisent par là même l'idée d'une procédure démocratique pure de tout engagement substantiel ${ }^{24}$. Waldron, par exemple, ne peut sans contradiction soutenir à la fois que l'égalité est un principe démocratique de base ${ }^{25}$ et que la majorité peut décider de tout. Même une conception soi-disant purement procédurale est obligée de reconnaître qu'il existe des valeurs fondamentales ou des principes constitutifs de la démocratie sur lesquels ses membres ne peuvent revenir, et qu'ils ne peuvent pas non plus fonder par le jeu démocratique normal. Tout n'est jamais entièrement laissé au libre choix de la majorité: à commencer par la détermination de la procédure de décision elle-même, certains choix doivent se faire indépendamment de la procédure afin de lui donner sens. C'est bien l'engagement préalable en faveur de l'égalité et de la liberté de choix qui conduit à définir une procédure de décision ouverte, dans laquelle chacun dispose d'un droit de vote égal: la détermination de ces valeurs est antérieure à l'application de la procédure, et n'est pas laissée à la libre appréciation des citoyens.

Au terme du raisonnement, il paraît bien logiquement impossible d'adhérer à une conception purement procédurale de la démocratie. Mais a-t-on par-là établi qu'une conception purement procédurale de la légitimité démocratique serait impossible ou contradictoire? Il semble qu'on pourrait très bien admettre que les procédures démocratiques reflètent certaines

24. Voir Dworkin, Une question de principe, p. 94.

25. "The Substance of Equality", Michigan Law Review, vol. 89, $\mathrm{n}^{\circ}$ 6, mai I99I, p. I359; "Constitutionalism: A Skeptical View", in Thomas Christiano et John Christman, dir., Contemporary Debates in Political Philosophy, Malden, Wiley-Blackwell, 2009, p. 274. 
valeurs fondamentales, sans suspendre la légitimité démocratique au fait que les résultats de ces procédures respectent certains critères substantiels. Selon cette vue, dans la mesure où elles procèdent de méthodes de décision respectueuses des citoyens en tant qu'agents moraux égaux, la décision d'augmenter l'impôt sur les sociétés, celle de permettre à des entreprises privées de contribuer au financement de campagnes électorales et celle d'interdire la réunion de certains groupes politiques seraient toutes également légitimes - d'une légitimité exclusivement procédurale.

Comme l'examen de ces exemples tend à le suggérer, toutefois, il n'est sans doute pas possible d'établir une séparation aussi étanche entre les valeurs posées ex ante et le contenu des décisions ex post. En effet, si les procédures se fondent sur certains principes substantiels constitutifs (les principes démocratiques fondamentaux d'égalité, de participation et d'inclusion), il semble naturel de pouvoir invoquer ces mêmes principes pour contester, au nom de la démocratie légitime, certaines décisions démocratiques formellement régulières mais dont la teneur les contredirait, ou bafouerait la conception des citoyens comme agents moraux égaux à laquelle ces principes se réfèrent. Cette remarque vaut au premier chef pour les décisions qui excluraient certaines personnes de la participation au processus démocratique de décision, ou créeraient des citoyens de seconde zone en comptant leur voix pour moins que celle des autres dans ce processus. À elle seule, cette concession suffit à disqualifier la thèse de la légitimité exclusivement procédurale des décisions démocratiques ${ }^{26}$ : la légitimité s'évalue aussi ex post, à l'aune de la conformité des décisions aux principes démocratiques fondamentaux. Mais rien n'empêche a priori d'élargir encore le champ d'application de ces principes substantiels: au vu de la généralité des concepts d'inclusion, de participation ou d'agentivité morale égale, il n'est pas absurde de se demander, par exemple, si le financement des campagnes électorales par des entreprises, voire une augmentation massive de l'impôt sur les sociétés, ne constituent pas des atteintes substantielles à ces principes démocratiques constitutifs. Quant à savoir s'il est possible de s'accorder sur les implications concrètes de ces principes et sur celles des décisions démocratiques qui les réalisent ou y contreviennent au contraire, c'est, bien sûr, une tout autre question.

26. C'est probablement la raison pour laquelle Waldron se refuse à une telle concession, maintenant, comme nous le mentionnions, que les membres d'une démocratie peuvent décider de tout, y compris de l'abolir ( Precommitment and Disagreement», in Larry Alexander, dir., Constitutionalism: Philosophical Foundations, Cambridge, Cambridge University Press, 200I, p. 272). Comme le souligne Thomas Christiano cependant, cette position est dépourvue de "cohérence normative" ("Waldron on Law and Disagreement", Law and Philosophy, vol. I9, $\mathrm{n}^{\circ} 4$, juillet 2000, p. 54I) : à partir du moment où l'autodétermination inclusive et égale est reconnue comme un principe constitutif de la démocratie, le choix démocratique d'abolir la démocratie ne devrait logiquement pas pouvoir être considéré comme démocratiquement légitime. 


\subsection{Approches purement substantielles}

L'impossibilité du pur procéduralisme ne laisse d'autre choix que d'envisager la démocratie à partir de certains principes substantiels constitutifs. En ce sens, l'adoption d'une conception substantielle de la démocratie, qui la définit par un ensemble de valeurs fondamentales ou de principes constitutifs, n'est pas une option: elle est inévitable, logiquement impliquée par les contradictions du pur procéduralisme. À première vue, une telle approche semble en outre pallier les insuffisances normatives qui s'attachent à la conception procédurale. Elle ne réduit pas la démocratie au processus électoral ou à des procédures de vote, mais fait pleinement droit à l'idée qu'une démocratie est une société démocratique, caractérisée par une certaine culture publique et attachée au respect et à la promotion d'un ensemble de principes politiques qui la définissent comme telle et en font un système doué de valeur. Les remarques précédentes suggèrent par ailleurs que cette compréhension substantielle de la démocratie s'arrime à une conception elle-même substantielle de la légitimité démocratique, d'après laquelle la démocratie est légitime si les décisions collectives qui y sont prises respectent et promeuvent, dans leur teneur, les principes démocratiques constitutifs. La réfutation logique du pur procéduralisme fournit ainsi des armes aux objections normatives qu'on peut par ailleurs lui opposer: si la démocratie se définit par le respect de certains principes, et si la procédure démocratique comme les résultats auxquels elle donne lieu sont évalués à l'aune de ces principes, il devient possible de tenir certaines décisions majoritaires pour tyranniques et de les contester comme telles.

Pour toutes ces raisons, l'approche substantielle est séduisante. Pourtant, de même que les approches purement procédurales de la démocratie et de la légitimité se révèlent impossibles ou contradictoires, on peut montrer qu'une approche purement substantielle de la démocratie déboucherait sur une impasse logique. La démonstration est même dans ce cas beaucoup plus rapide. Car que serait une conception purement substantielle de la démocratie et de la légitimité des décisions démocratiques? Pour le dire de manière délibérément brutale, ce serait une conception dans laquelle les citoyens ne pourraient jouer aucun rôle significatif. Si tous les principes démocratiques sont définis comme tels à l'avance, la libre détermination collective des citoyens est, au mieux, superflue, au pire, contre-productive. L'exercice d'autodétermination se bornerait à valider des principes préétablis, ou devrait être ignoré en cas de variation. La conception purement substantielle de la démocratie et de la légitimité démocratique, en d'autres termes, revient à nier l'effectivité des principes sur lesquels elle prétend se fonder.

Un point mérite d'être précisé. L'argument n'est pas ici qu'il est absurde de concevoir la légitimité politique en des termes purement substantiels. Il est plutôt que cette conception ne saurait s'appliquer au régime démocratique. Il est tout à fait possible, en effet, de considérer que ne sont légitimes que des décisions politiques justifiées, bonnes ou justes dans leur teneur, indépendam- 
ment de la manière dont elles ont été prises. Selon cette conception substantielle, une décision politique se juge à sa qualité propre (sa justice intrinsèque, ses heureuses conséquences, etc.), non à son auteur ou aux méthodes suivies pour la prendre. C'est par exemple la position de Joseph $\mathrm{Raz}^{27}$, qui soutient qu'un choix est légitime s'il est valable, peu importe que ceux à qui il s'applique l'approuvent ou non. Pour Raz, le «consentement» qui pourrait être exprimé par le biais du vote ne contribue en aucune façon à fonder la légitimité des décisions politiques; une décision légitime est, en tout et pour tout, une décision justifiée. La correction substantielle des mesures politiques est tenue pour la condition nécessaire et suffisante de leur légitimité.

Quels que soient les mérites de cette conception, il semble assez évident qu'elle n'est défendable que dans un cadre extra-démocratique, comme dans le cas d'une sentence judiciaire ou des instructions d'un maître d'école (encore que, là aussi, le consentement des tiers ou la procédure suivie pour prendre les décisions concoure, semble-t-il, à leur légitimité). Il paraît logiquement impossible d'appliquer le principe d'une légitimité exclusivement substantielle des décisions politiques à la démocratie, sauf à travestir complètement la signification historique et ordinaire de ce concept. Un régime dont les membres ne disposeraient d'aucun contrôle sur la façon dont sont menées les affaires communes ne serait pas reconnu comme démocratique. Ce contrôle peut passer, entre autres, par la participation directe aux décisions publiques, par la délégation de responsabilités décisionnelles et leur évaluation périodique par le vote, par l'exercice d'une supervision plus diffuse et l'accès à divers canaux de contestation et de critique de l'action politique ${ }^{28}$. On peut déplorer, avec certains observateurs, que ce contrôle soit réduit à la portion congrue dans les démocraties existantes. Il demeure qu'il exprime un impératif inhérent au système démocratique: que les citoyens aient un droit de regard sur les lois et mesures politiques qui les obligent. Les décisions prises dans l'irrespect de cet impératif démocratique sont soupçonnées d'être illégitimes. En ce sens, une conception de la démocratie et de la légitimité qui se voudrait purement substantielle est une contradiction dans les termes.

On voit aisément pourquoi, plus précisément, l'application d'une logique razienne au contexte démocratique débouche sur une contradiction. $\mathrm{Si}$, pour être démocratique et légitime, une décision n'avait strictement qu'à se conformer à des principes substantiels prédéfinis (respect des droits de l'homme, réalisation de l'égalité sociale, encouragements au plein dévelop-

27. "Facing Diversity: The Case of Epistemic Abstinence", Philosophy and Public Affairs, vol. I9, n I, janvier I990, p. 46; The Morality of Freedom, Oxford, Oxford University Press, I988, p. 37; voir aussi Richard J. Arneson, "The Supposed Right to a Democratic Say ", in Thomas Christiano et John Christman, dir., Contemporary Debates in Political Philosophy, Malden, Wiley-Blackwell, 2009, p. I95-2I2.

28. Voir Philip Pettit, "Democracy, Electoral and Contestatory», in Ian Shapiro, et Stephen Macedo, dir., Designing Democratic Institutions, New York, New York University Press, 2000, p. I05-I 44 (NOMOS XLII). 
pement de soi, par exemple), comme le soutient la conception substantielle, alors il n'importerait pas de savoir qui prend la décision. Suivant cette logique, ou pour la pousser à l'absurde, on pourrait à la rigueur tenir pour démocratiquement légitime un régime dans lequel une seule personne prendrait toutes les décisions politiques pour toutes les autres, tant que ces décisions se conformeraient, dans leur teneur, aux valeurs prédéfinies comme démocratiques. C'est là la conséquence aberrante de la thèse selon laquelle la légitimité démocratique est exclusivement affaire de justification substantielle, et pas du tout de consentement. On pourrait bien entendu objecter, à raison, que les principes constitutifs de la démocratie impliquent que les citoyens participent à l'élaboration des décisions politiques et excluent, par conséquent, de confier la décision à un seul. La conception substantielle de la légitimité démocratique requiert une implication des citoyens dans la confection des lois qui les lient.

Mais on ne ferait ainsi que mettre en évidence une nouvelle contradiction de toute conception purement substantielle de la démocratie. Car si les principes démocratiques eux-mêmes impliquent de laisser aux citoyens la charge de se déterminer - c'est-à-dire, s'ils ménagent dans le concept de démocratie une part d'indétermination - alors une conception purement substantielle, qui équivaut à une conception intégralement déterminée de la démocratie, revient tout bonnement à nier les principes démocratiques qu'elle devrait intégrer.

Il est donc bien évident qu'aucun théoricien n'adopte à proprement parler de conception purement substantielle de la démocratie. Pour pouvoir défendre une conception plausible, ou au moins logiquement possible, de la démocratie, les partisans du substantialisme sont forcés d'intégrer la considération des procédures dans leur démonstration à un moment ou à un autre. La conception de Ronald Dworkin, par exemple, qui représente en un sens la défense la plus systématique d'une approche substantielle de la démocratie et de la légitimité2 ${ }^{2}$, admet la participation politique au rang des conditions constitutives de la démocratie ${ }^{30}$. Or, soutenir que la conception substantielle de la légitimité démocratique exige entre autres choses que les citoyens aient une «part égale» dans l'élaboration des lois qui les lient, c'est reconnaître que le concept de démocratie implique, sinon des procédures de décision collective absolument déterminées, du moins un ensemble possible de procédures qui satisfasse ce «principe de participation» fondamental. En ce sens, l'affirmation de Dworkin selon laquelle la «démocratie est une forme de gouvernement procéduralement incomplète ${ }^{31}$ » est en partie contes-

29. La vertu souveraine, Bruxelles, Émile Bruylant, 2007, p. 3 I9.

30. «Equality, Democracy, and Constitution: We the People in Court ", Alberta Law Review, vol. 28, $\mathrm{n}^{\circ} 2$, janvier I990, p. 338 ; Freedom's Law: The Moral Reading of the American Constitution, Cambridge, Oxford University Press, I996, p. 24.

31. Freedom's Law, p. 33. 
table. Elle est vraie, dans la mesure où la démocratie ne souffre pas de définition exclusivement procédurale et s'accommode par ailleurs probablement de différentes procédures de décision (c'est-à-dire, les tient pour également légitimes). Elle peut être sérieusement mise en doute, en revanche, si elle signifie que le choix de la procédure de décision ne doit s'appuyer que sur les performances de celle-ci (la qualité des résultats qu'elle produit), et non sur les valeurs démocratiques que sa mise en œuvre permet, ou non, de réaliser. En d'autres termes, le fait que la démocratie s'appuie sur certains types de procédures de décision de préférence à d'autres (discriminatoires, inégalitaires ou excluantes) renvoie bel et bien à un trait constitutif de la démocratie, et le manquement à ces procédures constitue une marque d'illégitimité spécifiquement démocratique.

L'analyse des conceptions exclusives de la légitimité aboutit donc à deux conclusions symétriques. D'une part, la critique des conceptions purement substantielles a fait apparaître qu'il n'est pas possible d'évacuer entièrement la dimension procédurale de la légitimité démocratique. Le respect de certaines procédures de décision se révèle un élément incontournable, insubstituable, de la légitimité politique en démocratie. D'autre part, la critique des conceptions purement procédurales a montré qu'aucune théorie de la légitimité démocratique ne peut faire l'économie de certaines valeurs substantielles, constitutives de l'idéal démocratique, non seulement pour déterminer ses procédures, mais, dans une certaine mesure, pour évaluer ses résultats. Il en ressort que, pour être viable, toute conception de la légitimité démocratique doit nécessairement être mixte ou duale.

De fait, de même que Waldron renonce sans l'avouer à son pur procéduralisme en reconnaissant l'égalité de statut comme une valeur démocratique fondatrice, Dworkin est contraint d'accorder une valeur relative à la participation politique pour penser la démocratie. En fait de conceptions "pures» de la démocratie et de la légitimité, ce sont autrement dit des conceptions mixtes, à dominante soit procédurale, soit substantielle, qui s'affrontent dans la littérature contemporaine. Le défaut de ces différentes conceptions est ainsi moins, paradoxalement, leur posture "puriste» ou exclusive, que le fait que leur mixité n'est pas assumée. Elles sous-théorisent, quand elles ne le nient pas, le rôle de la composante adverse qu'elles sont contraintes de réintégrer en sous-main. Par-là, elles s'interdisent de penser l'articulation des composantes procédurales et substantielles, et exposent l'appréciation de la légitimité démocratique à une instabilité permanente. Le reste de cet article se propose donc d'examiner ce que pourrait être une conception duale assumée, et s'il est possible d'y articuler les exigences procédurales et substantielles d'une façon plus satisfaisante.

\section{Dualité et priorité}

L'impasse des conceptions procédurales et substantielles de la démocratie et de la légitimité démocratique dans leur version moniste conduit à les envi- 
sager, non pas comme des approches complètes et opposées, mais comme l'expression d'exigences démocratiques distinctes, que toute théorie normative devrait s'efforcer de satisfaire. On peut ainsi poser que, pour être légitime, la démocratie doit à la fois reposer sur un système populaire et égalitaire de détermination des décisions communes, qui assure que les règles destinées à valoir pour tous recueillent l'assentiment de tous, et répondre (au moins) aux exigences substantielles exprimées par les idéaux de participation, d'égalité et d'inclusion en se gouvernant selon des lois justifiées, raisonnables ou justes. Une conception satisfaisante de la légitimité démocratique doit essayer de tenir ensemble les pôles du consentement et de la validité, de la volonté et de la justification.

Bien entendu, cette conception duale de la légitimité se révèle "particulièrement problématique ${ }^{32}$ » dès le moment où elle s'énonce, puisque se pose immédiatement la question de savoir ce qu'il advient lorsque ces différents éléments entrent en contradiction. Faut-il tenir les deux composantes pour des conditions également nécessaires de la légitimité d'une décision, et rejeter comme illégitimes toutes celles qui conviendraient soit à l'une, soit à l'autre? Dans la mesure où l'illégitimité est un concept pratique, qui commande notamment certaines attitudes de la part des citoyens (telles que l'obéissance aux lois et le droit, voire le devoir, de résistance), cette première position peut paraître démesurément exigeante et entraîner en soi des conséquences anarchiques généralisées qu'une conception viable de la légitimité politique devrait plutôt chercher à circonscrire. Mais si l'on admet que, pour une décision donnée, les deux exigences n'ont pas nécessairement à être également satisfaites, comment les considérations procédurales et substantielles devront-elles être ordonnées en cas de conflit entre les deux dimensions de la légitimité démocratique ? Faut-il passer outre la décision résultant d'une procédure démocratique régulière? Ou tenir pour légitime une loi inique ? Est-il légitime par exemple, au regard de la conception duale avancée ici, que la communauté démocratique fasse passer une loi rendant plus difficile l'exercice du droit de vote pour les Noirs ou les pauvres? Ou encore, qu'elle vote une réforme fiscale faisant intégralement porter la charge de l'impôt sur une seule catégorie de la population? Une conception duale ne fournit a priori pas de raison de tenir les questions substantielles de justice et de droit pour plus ou moins importantes, dans l'évaluation de la légitimité des décisions communes, que la conformité de celles-ci à certaines exigences d'égalité ou d'inclusion de la procédure ${ }^{33}$. Or, en l'absence d'une telle raison,

32. Beitz, Political Equality, p. 48 ; voir aussi Elizabeth Anderson, «The Epistemology of Democracy", Episteme, vol. 3, n I, 2006, p. I I ; José Luis Martí, «The Epistemic Conception of Deliberative Democracy Defended ", in Samantha Besson et José Luis Martí, dir., Deliberative Democracy And Its Discontents: National and Post-National Challenges, Burlington, Ashgate Publishing, 2006, p. 37.

33. Voir Thomas Christiano, "The Authority of Democracy", Journal of Political Philosophy, vol. I2, $\mathrm{n}^{\circ} 3,2004$, p. 269. 
la conception duale de la légitimité démocratique menace d'imploser à la première tension entre les exigences procédurales et substantielles. Il ne suffit pas par conséquent que cette conception émerge des ruines logiques de ses concurrentes monistes. Pour être plausible, elle doit encore montrer comment les exigences procédurales et substantielles peuvent être conciliées, et ordonnées en cas de conflit.

\subsection{La démocratie au long cours}

La première proposition que nous voudrions avancer pour progresser dans ce sens est que la conciliation entre exigences procédurales et substantielles est plus facilement envisageable dès lors que l'on aborde la démocratie, ou la prise de décision en démocratie, depuis une perspective temporelle spécifique. Plusieurs de philosophes politiques ont récemment invité à troquer la conception statique de la démocratie comme simple procédure de décision contre une conception plus «dynamique ${ }^{34} »$. Selon celle-ci, la démocratie opère comme un processus continu d'autodétermination et, par conséquent, ne condense pas toute sa légitimité dans le verdict d'une procédure ponctuelle de choix. Dans le cadre théorique esquissé par cet article, on peut alors se représenter la démocratie comme une entreprise historique de clarification, par la communauté démocratique elle-même, des implications politiques concrètes des principes qui la constituent. La démocratie est moins une procédure qu'un processus «en cours» ou «au long cours » ${ }^{35}$, par lequel se définit, s'accumule, mais se révise aussi, un fond substantiel de décisions démocratiques sur les principes qui organisent la vie commune.

Héritée du procéduralisme et, avant lui, de la théorie du choix social, la perspective statique sur la démocratie est réductrice et insatisfaisante, parce qu'elle ne permet pas de penser la continuité de la politique démocratique. Identifier la démocratie à une procédure discrète de détermination de la décision collective, cela revient à tenir pour rien ce qui se passe dans l'intervalle entre deux décisions politiques et à s'interdire de comprendre la façon dont ces décisions se rapportent les unes aux autres. Un trait important de l'organisation démocratique est, en effet, que les mesures politiques y sont discutées, arrêtées, testées et discutées à nouveau, et que les décisions ultérieures se prennent à la lumière de celles auxquelles la discussion avait provisoirement abouti. Les décisions collectives (les élections, les lois) apparaissent ainsi comme les moments d'un processus qui ne prend pas fin une fois la loi votée ou l'élection passée, mais qui se poursuit dans le temps et fait le lien entre les différentes décisions prises.

34. Voir par exemple Anderson, "The Epistemology of Democracy ", p. 222; Hélène Landemore, Democratic Reason: Politics, Collective Intelligence, and the Rule of the Many, Princeton, Princeton University Press, 20I 2, p. I7, 22.

35. James Bohman, «Epistemic Value and Deliberative Democracy », The Good Society, vol. I8, n 2, 2009, p. 30-3I. 
Depuis la perspective statique, le vote est censé signer la fin de la réflexion de la communauté démocratique sur une question donnée; la décision majoritaire est présentée comme le dernier mot de la discussion démocratique sur le sujet. Pourtant, le fait que la minorité persiste dans son opposition de fond à la décision (tout en en reconnaissant la force obligatoire) suggère que la détermination de la volonté majoritaire ne marque bien souvent qu'un terme provisoire dans le processus d'autodétermination démocratique. Une fois la décision prise ou l'élection passée, l'opposition continue de produire des raisons à l'appui de sa position ${ }^{36}$, et cherche à y rallier d'autres membres de la communauté démocratique. Toute décision majoritaire se présente ainsi comme une conclusion temporaire, que l'on peut présumer valide "jusqu'à ce que de bonnes raisons [...] viennent la contester $^{37}$ ». Le processus démocratique ayant conduit à autoriser le mariage entre personnes du même sexe en France illustre bien cette logique de consolidation progressive. Lors de l'instauration du pacte civil de solidarité (Pacs) en I999, les arguments en faveur du mariage pour tous n'étaient visiblement pas assez développés ou n'avaient pas reçu suffisamment d'écho pour obtenir le soutien de la majorité, qui s'était contentée d'autoriser une forme plus souple et moins protectrice d'union civile. Loin de clore le sujet, l'application de la loi instaurant le Pacs a été l'occasion de controverses continues entre adversaires de la loi et partisans de son approfondissement, qui ont abouti à l'adoption en $20 \mathrm{I}_{3}$ de la loi sur le mariage pour tous. Au vu des oppositions qu'elle a fédérées contre elle et des nombreux débats qu'elle a fait naître (sur la gestation pour autrui notamment), on peut supposer que cette loi n'est à son tour qu'une conclusion provisoire dans un processus en cours.

Dans la perspective dynamique, autrement dit, les procédures démocratiques de décision sont réputées faillibles et acceptées comme telles. Or cela lui confère un avantage certain sur la perspective statique, lorsqu'il s'agit de juger de la légitimité des décisions démocratiques. Cette dernière suppose en effet d'évaluer la légitimité démocratique de façon discrète: si la démocratie est une suite de décisions discontinues, alors il est exigé de chaque décision qu'elle soit pleinement légitime. Or, couplée à la conception duale de la légitimité que nous cherchons à défendre, cette perspective est tout bonnement intenable, car il est impossible que chaque décision démocratique soit procéduralement et substantiellement sans reproche ${ }^{38}$. Plus

36. Bernard Manin, "Volonté générale ou délibération ? Esquisse d'une théorie générale de la délibération politique ", Le Débat, vol. 33, 1985, p. 72-93.

37. Seyla Benhabib, "Toward a Deliberative Model of Democratic Legitimacy", in Seyla Benhabib, dir., Democracy and Difference: Contesting the Boundaries of the Political, Princeton, Princeton University Press, 1996, p. 72; voir aussi Jürgen Habermas «Reply to Symposium Participants ", Cardozo Law Review, vol. I7, I996, p. I494.

38. C'est sans doute pourquoi ce sont généralement les conceptions purement procédurales de la démocratie qui adoptent la perspective statique. 
précisément, si la conception duale devait signifier que chaque décision démocratique doit, pour être légitime et obligatoire, satisfaire les exigences procédurales et substantielles de la légitimité, elle serait à peu près irréalisable, et de surcroît invérifiable. Il est manifestement impossible d'obtenir que tous s'accordent sur la justice ou la supériorité substantielle d'une décision particulière, contre laquelle un certain nombre d'entre eux viennent précisément de se prononcer; et il n'est pas non plus envisageable de vérifier, à chaque occurrence, que la décision prise est effectivement celle qui est la plus conforme aux principes constitutifs de la communauté démocratique. S'il pouvait en être autrement dans l'un ou l'autre cas, les membres de cette communauté n'auraient nul besoin de recourir au vote pour se déterminer. Dans un contexte où l'accord sur les implications précises des principes démocratiques fondamentaux est, au mieux, tendanciel, et toujours imparfaitement réalisé, suspendre le caractère obligatoire de chaque décision collective à sa conformité effective à ces principes (ou, d'ailleurs, aux exigences de la justice ou du «bien commun») aurait des conséquences anarchiques ruineuses pour toute théorie de la légitimité.

Réinscrire la légitimité dans le contexte d'une démocratie au long cours permet en ce sens de tempérer ce qui pourrait apparaître comme une exigence démesurée de la conception duale. En abordant la décision majoritaire comme une détermination temporaire, susceptible d'être révisée, on reconnaît à la communauté démocratique un droit à l'erreur. Un tel droit signifie proprement que la communauté démocratique peut ponctuellement prendre de mauvaises décisions politiques - c'est-à-dire, des décisions qui ne remplissent pas les conditions substantielles de la légitimité démocratique - , sans que cela rende pour autant nécessairement cette décision illégitime ou lui ôte sa valeur d'obligation. Dans la perspective d'une démocratie au long cours, les deux composantes de la légitimité peuvent être prises en considération sans que la valeur obligatoire des décisions démocratiques soit systématiquement suspendue à l'attestation de leur validité substantielle.

Il convient de souligner qu'en comprenant la démocratie comme régime dynamique, qui tendrait par sa double capacité d'accumulation et de révision à de meilleures décisions politiques et à une approximation des justes principes de la communauté démocratique, la conception duale défendue ici se rattache aux approches dites «épistémiques» de la légitimité démocratique. Selon ces approches, la démocratie tire sa légitimité de ce qu'elle est plus susceptible que d'autres régimes d'aboutir, de façon générale, à des résultats politiques de qualité, justes ou justifiés. Différents éléments peuvent être avancés pour étayer la thèse de la supériorité épistémique de la démocratie. On peut notamment faire valoir que les propriétés spécifiques des procédures démocratiques, le fait qu'elles permettent la participation égale d'un grand nombre de personnes diverses à la décision, mais également qu'elles soient récursives et incluent la possibilité d'une réévaluation des décisions passées, contribuent à la prise de meilleures décisions poli- 
tiques à long terme. Il est fort probable que d'autres conditions sociales doivent être remplies pour que le pari épistémique d'une coïncidence tendancielle des exigences procédurales et substantielles de la légitimité démocratique soit tenable. Leur examen, cependant, dépasse le cadre de ce papier.

Interprétée dans une perspective dynamique, la conception duale nous offre donc une conception beaucoup plus souple, et réalisable, de la légitimité démocratique: les critères procéduraux et substantiels n'ont pas besoin d'être satisfaits conjointement de façon constante pour que la démocratie soit légitime. Dire cela n'implique pas, il faut le souligner, de rabattre la légitimité de la décision sur l'une de ses composantes. La conception de la légitimité mise en avant ici est bien duale, en ce que les deux critères doivent être remplis, c'est-à-dire qu'ils constituent tous deux des conditions nécessaires, et également nécessaires, de la légitimité démocratique. Seulement, c'est l'incapacité durable ou tendancielle à les respecter, et non l'échec ponctuel d'une décision commune à les réaliser, qui rend la démocratie illégitime.

\subsection{Une priorité lexicale}

Plus précisément, dans la conception que nous défendons ici, c'est l'exigence substantielle de qualité des décisions démocratiques qui peut occasionnellement ne pas être satisfaite sans que cela nuise à la légitimité démocratique de l'ensemble. Les exigences procédurales, elles, doivent être respectées en chaque occurrence. Les principes d'égalité, de participation et d'inclusion sur lesquels reposent ces exigences procédurales excluent d'adopter un certain nombre de méthodes de décision (celle qui confierait les décisions collectives aux plus intelligents, aux plus jeunes ou aux dieux, par exemple), mais le spectre des procédures démocratiques légitimes n'en demeure pas moins relativement varié. Dire que chaque décision démocratique doit satisfaire les exigences procédurales ne signifie donc évidemment pas que toutes les décisions communes devraient être prises par référendum populaire, à la majorité des voix égales, pour que la démocratie puisse prétendre à la légitimité. L'autodétermination collective exige que chaque citoyen dispose d'un certain contrôle égal sur les décisions collectives, et celle-ci peut en ce sens parfaitement s'incarner dans un système de décision à plusieurs vitesses visant à protéger, par exemple, certains droits considérés comme fondamentaux des revirements d'une majorité simple. De même, cela ne signifie pas que le moindre vice de procédure suffit à disqualifier une décision comme illégitime. L'idée est plutôt qu'aucune décision politique qui passerait outre les multiples formes que le contrôle démocratique peut prendre à l'intérieur d'un système institutionnel complexe ne pourrait prétendre à la légitimité démocratique.

Cela nous conduit à notre deuxième proposition: celle d'une priorité lexicale de la composante procédurale sur la composante substantielle de la légitimité démocratique. Nous empruntons le concept de John Rawls, qui 
défend la thèse d'une "priorité de la liberté» sur le principe de différence ${ }^{39}$. L'idée générale est la même: la "part égale» que garantissent de façon insubstituable les procédures d'autodétermination inclusive et égale ne peut être sacrifiée à la justesse des résultats démocratiques d'ensemble ${ }^{40}$. Dans notre cas, cette priorité lexicale signifie plus précisément que, tandis que les décisions démocratiques peuvent ponctuellement déroger aux exigences substantielles de justification sans cesser pour autant d'être légitimes, la violation des exigences générales d'inclusion, de participation et d'égalité exprimées par les procédures démocratiques de décision suffit en revanche à ôter aux décisions politiques leur légitimité démocratique, quelle que soit par ailleurs leur teneur. Elle n'implique en revanche nullement que la participation égale de tous à la détermination des décisions communes suffise à asseoir la légitimité du régime démocratique. En effet, que celui-ci démontre une tendance dynamique à produire une législation conforme aux principes démocratiques constitutifs (dans l'extension variable qu'on peut donner à leur champ d'application) demeure une condition nécessaire de sa légitimité. De même, si l'on veut, que la priorité de la liberté posée par les partenaires rawlsiens dans la théorie de la justice comme équité ne signifie pas que le principe de liberté soit le tout de la justice, puisque le respect du principe de différence représente une condition également nécessaire de sa réalisation, de même la priorité des exigences procédurales n'implique pas que leur respect suffise à fonder la légitimité de la politique démocratique. Sans tendance dynamique à produire des décisions conformes aux valeurs fondamentales de la communauté politique, les procédures d'autodétermination inclusive et égale ne sont pas pleinement légitimes.

La nécessité d'adopter une conception duale de la légitimité démocratique s'impose en vertu de l'impossibilité logique des approches exclusives. De façon comparable, l'hypothèse d'une priorité lexicale de la composante procédurale tire sa plausibilité de la déroute des hypothèses concurrentes. Imaginons en effet que nous accordions une priorité lexicale à la substance plutôt qu'à la procédure, ou encore que nous refusions d'assigner une priorité définitive à l'une ou à l'autre des composantes. Cette dernière solution est défendue par Eric MacGilvray ${ }^{41}$, qui préconise d'évaluer intuitivement la légitimité de chaque décision démocratique en mettant en balance les gains et les pertes respectivement associés au sacrifice de l'une ou l'autre composante dans le cas donné. Ces propositions se heurtent à une objection identique: qui sera juge du caractère substantiellement inacceptable de la décision collec-

39. Théorie de la justice, Paris, Seuil, 2009, p. 68-69.

40. L'analogie avec le raisonnement de Rawls ne va cependant pas plus loin, car les principes de la théorie rawlsienne de la justice n'ont rien de commun avec les composantes de la légitimité dont il est question ici, non plus que les raisons respectivement avancées pour justifier leur mise en ordre lexical.

41. "Democratic Doubts: Pragmatism and the Epistemic Defense of Democracy », Journal of Political Philosophy, vol. 22, $\mathrm{n}^{\circ} \mathrm{I}$, mars 20I4, p. II 5-I 20. 
tive? On retrouve là un argument déjà mobilisé contre les approches statiques de la démocratie: les propositions substantielle et intuitionniste débouchent sur une impasse, parce qu'il n'est pas possible, ponctuellement et en chaque cas, de déterminer quelle est assurément la bonne décision. Celle-ci peut se révéler telle a posteriori, une fois ses conséquences éprouvées, ou les implications concrètes des principes démocratiques s'approcher sur le long terme, mais il semble très difficile, voire impossible, de les établir avec certitude sur le moment, et surtout en chaque cas. S'engager à mettre à chaque fois en balance la source procédurale des décisions avec leurs vertus substantielles, c'est éternellement reconduire le désaccord premier autour de ce qui doit faire autorité en démocratie - c'est, comme le dit Waldron, poser à neuf le problème que la décision collective avait pour objet de régler. En affirmant, au contraire, qu'en cas de conflit entre les composantes procédurales et substantielles de la légitimité, le respect de la procédure démocratique doit primer, on dispose d'une règle de priorité qui neutralise ces implications anarchiques, sans pour autant achever les disputes quant à la justesse de la décision collective.

\section{Conclusion}

La conception duale de la légitimité démocratique que nous avons tenté de défendre repose, encore une fois, sur un présupposé majeur, celui de la capacité effective du régime démocratique à aboutir, sur le temps long et de façon tendancielle, à un ensemble de bonnes décisions politiques. Si le régime démocratique ne manifeste pas de tendance dynamique et régulière à produire des résultats politiques qui respectent et promeuvent effectivement les principes démocratiques fondamentaux, alors il n'est pas légitime, même si ces résultats politiques procèdent de modes de décision inclusifs, égalitaires et participatifs. Or le cadre théorique que nous avons esquissé ici n'a fourni que peu d'éléments qui permettraient de penser qu'une telle tendance dynamique est plausible ou probable. Pour qu'elle soit pleinement défendable, il manque ainsi à notre conception duale d'être systématiquement articulée à une approche épistémique plus globale de la démocratie. Les raisons et conditions, parfois fort exigeantes, sous lesquelles le pari épistémique peut s'avérer rationnel, doivent faire l'objet d'un traitement séparé. 